\title{
Simulation of angiogenesis and cell differentiation in a CaP scaffold subjected to compressive strains using a lattice modeling approach
}

\author{
Clara Sandino ${ }^{a}$, Sara Checa ${ }^{b}$, Patrick J. Prendergast ${ }^{b}$, Damien Lacroix ${ }^{a, *}$ \\ ${ }^{a}$ Biomechanics and Mechanobiology, Institute for Bioengineering of Catalonia, C Baldiri Reixac, 4, Barcelona 08028, Spain \\ ${ }^{\mathrm{b}}$ Trinity Centre for Bioengineering, School of Engineering, Parsons Building, Trinity College, Dublin 2, Ireland
}

a b s t r a c t

Mechanical stimuli are one of the factors that influence tissue differentiation. In the development of biomaterials for bone tissue engineering, mechanical stimuli and formation of a vascular network that transport oxygen to cells within the pores of the scaffolds are essential. Angiogenesis and cell differentiation have been simulated in scaffolds of regular porosity; however, the dynamics of differentiation can be different when the porosity is not uniform. The objective of this study was to investigate the effect of the mechanical stimuli and the capillary network formation on cell differentiation within a scaffold of irregular morphology. A porous scaffold of calcium phosphate based glass was used. The pores and the solid phase were discretized using micro computed tomography images. Cell activity was simulated within the interconnected pore domain of the scaffold using a lattice modeling approach. Compressive strains of 0.5 and $1 \%$ of total deformation were applied and two cases of mesenchymal stem cells initialization ( in vitro seeding and in vivo) were simulated. Similar capillary networks were formed independently of the cell initialization mode and the magnitude of the mechanical strain applied. Most of vessels grew in the pores at the periphery of the scaffolds and were blocked by the walls of the scaffold. When $0.5 \%$ of strain was applied, $70 \%$ of the pore volume was affected by mechano-regulatory stimuli corresponding to bone formation; however, because of the lack of oxygen, only $40 \%$ of the volume was filled with osteoblasts. $40 \%$ of volume was filled with chondrocytes and $3 \%$ with fibroblasts. When the mechanical strain was increased to $1 \%, 11 \%$ of the pore volume was filled with osteoblasts, $59 \%$ with chondrocytes, and $8 \%$ with fibroblasts. This study has shown the dynamics of the correlation between mechanical load, angiogenesis and tissue differentiation within a scaffold with irregular morphology.

\section{Introduction}

Bone is a living tissue that under normal conditions regenerates by itself. Nevertheless, when a defect exceeds a critical size, bone regeneration can be induced through the implantation of a biomaterial in the diseased or damaged tissue region. This biomaterial serves as a scaffold for cells to migrate, proliferate, differentiate and synthesize new extracellular matrix. Within the characteristics of engineered scaffolds, the architecture has an important role. An appropriate porosity and interconnectivity are necessary to allow cells to enter into the scaffold and to promote the formation of a vascular network (angiogenesis); which supply cells with oxygen and nutrients essential for their survival. In addition, the mechanical environment plays a critical role in the biological

\footnotetext{
* Corresponding author. Tel.: p 34 934020266; fax: p 34934037181. E-mail address: dlacroix@ibec.pcb.ub.es (D. Lacroix).

URL: http://www.biomechanics.es
}

process. The scaffold mechanical properties need to be adequate to withstand the mechanical loads at the implantation site and to transmit the appropriate mechanical stimuli to the cells so that they follow the desired differentiation pathway.

Several mechanoregulation theories relate the magnitude of stress and strain present at the extracellular matrix and the interstitial fluid velocity with the formation of bone, cartilage and/or fibrous tissue [1-3]. Computational models based on Prendergast et al. [3] theory have successfully simulated the time course of tissue regeneration during fracture healing [4-7]. Additionally, this theory has been used to predict tissue growth at bone/implant interfaces [8], in bone chambers $[9,10]$ and in scaffolds for osteochondral defect healing [11]. All these models used a diffusion equation to describe the migration and proliferation of cells within the regenerating tissues, assuming that cells attempt to reach a uniform distribution. A random walk model was introduced by Perez and Prendergast [12] to model specific cell proliferation using a lattice modeling approach. This concept was used by Byrne et al. [13] to simulate tissue growth within a regular scaffold, in order to 

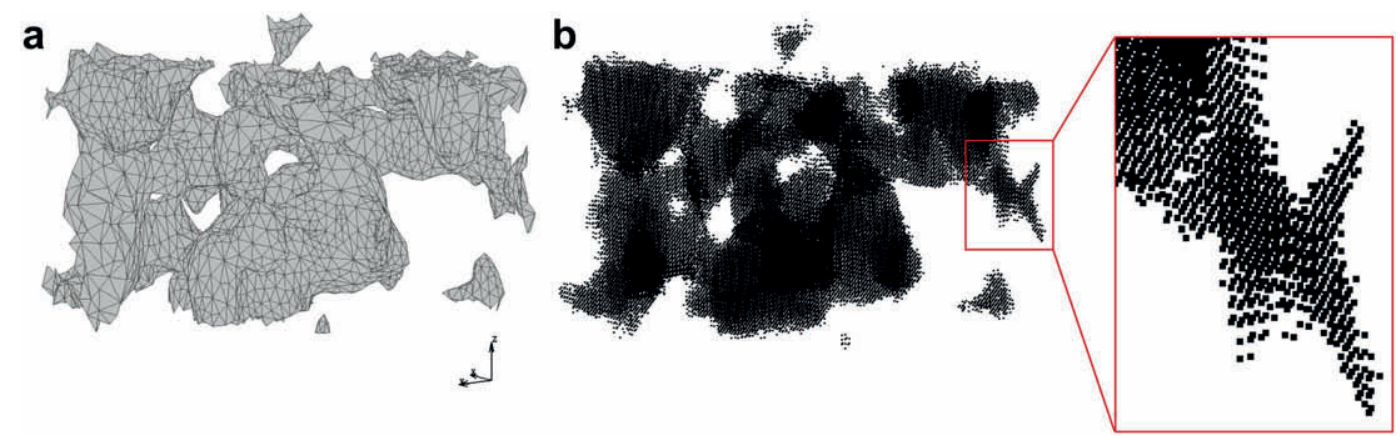

Fig. 1. Section of the scaffold used for the simulations. (a) Mesh of the interconnected pores used for FE models. (b) Lattice of the interconnected pores used for the cell activity simulation.

investigate the effect of scaffold design variables like porosity, Young's modulus and dissolution rate, and by Khayyeri et al. [14] to study tissue differentiation in an in vivo bone chamber.

In order to account for the role of angiogenesis during tissue differentiation, Geris et al. [15] developed a mathematical model to simulate tissue differentiation where cell density is regulated by the concentration of angiogenic factors. Checa and Prendergast [16] proposed a mechanobiological model to simulate capillary network formation and its effect on tissue growth in a bone/ implant interface using the lattice modeling approach. This model

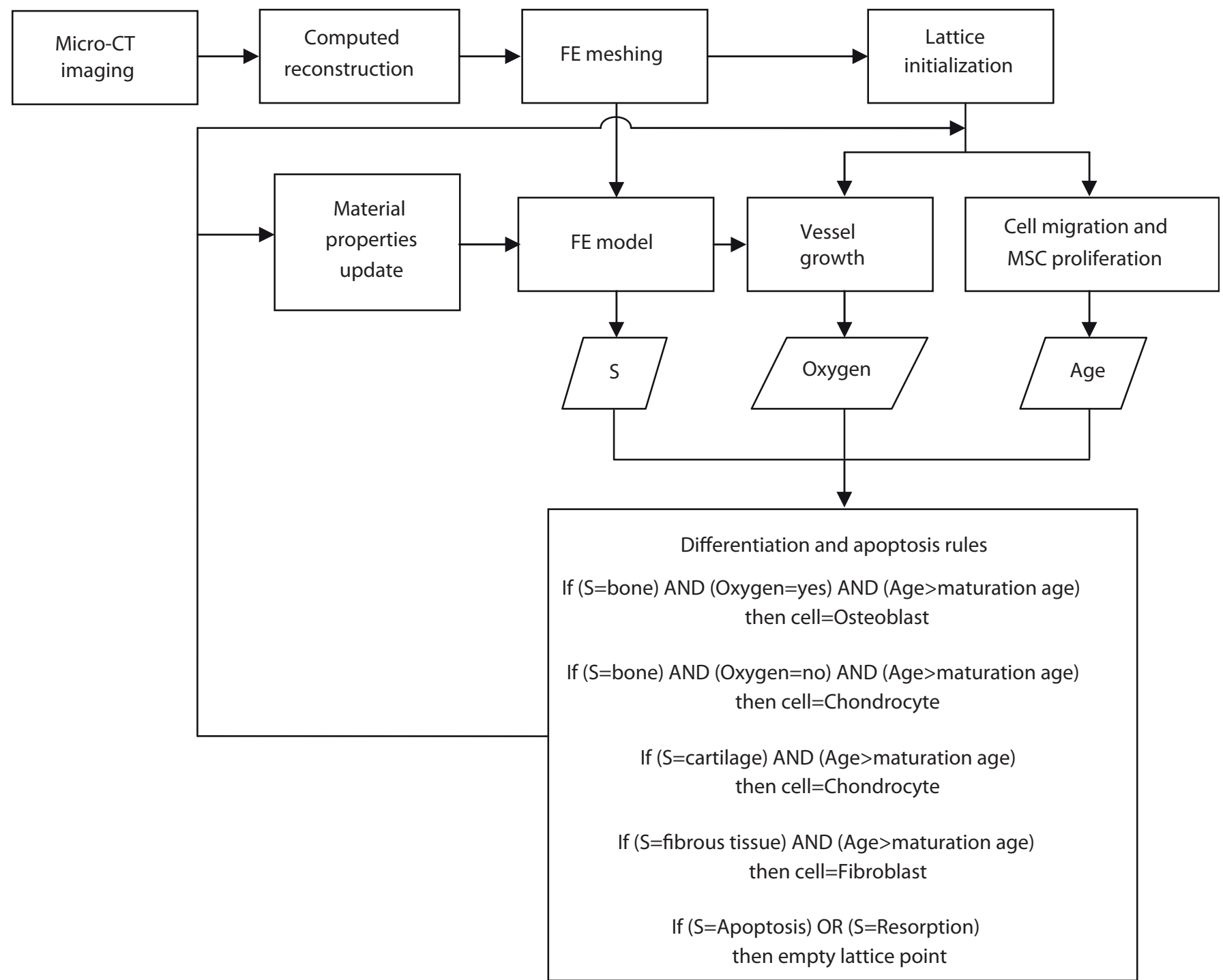

Fig. 2. Computational algorithm used to model cell differentiation and angiogenesis. The simulation of vessel growth, cell migration, MSC proliferation, MSC differentiation and cell apoptosis was performed in the lattice points within the interconnected pores of the scaffold. The mechano-regulatory stimuli $\quad \mathrm{S}$ affecting cells were computed using a finite element model. Mature MSCs differentiated according to $\mathrm{S}$ and oxygen supply. Material properties for the FE model were updated according to cell differentiation. The simulations finished when homeostasis was achieved. 
Table 1

Algorithm of cell differentiation and angiogenesis parameters.

\begin{tabular}{|c|c|c|c|}
\hline Cell type & Process & Probability/rate/frequency & Reference \\
\hline MSCs & In vivo initialization & 0.3 & Checa and Prendergast [17] \\
\hline MSCs & In vitro seeding initialization & $\mathrm{N}(1.5,0.45)$ & \\
\hline MSCs & Differentiation & 0.3 & Isaksson et al. [31] \\
\hline Osteoblasts & Apoptosis & 0.15 & Isaksson et al. [31] \\
\hline Chondrocytes & Apoptosis & 0.1 & Isaksson et al. [31] \\
\hline Fibroblasts & Apoptosis & 0.05 & Isaksson et al. [31] \\
\hline MSCs & Proliferation & 0.6 & Isaksson et al. [31] \\
\hline Osteoblasts & Proliferation & 0.3 & Isaksson et al. [31] \\
\hline Chondrocytes & Proliferation & 0.2 & Isaksson et al. [31] \\
\hline \multirow[t]{2}{*}{ Fibroblasts } & Proliferation & 0.55 & Isaksson et al. [31] \\
\hline & Oxygen diffusion distance & $100 \mathrm{~mm}$ & Carmeliet and Jain [32] \\
\hline MSCs Fibroblasts & Migration rate & $30 \mathrm{~mm} / \mathrm{h}$ & Appedu and Shur [33] \\
\hline $\mathrm{ECs}$ & Initialization & 0.1 & Checa and Prendergast [16] \\
\hline ECs & Vessel branching & $\begin{array}{cc}0 & \text { ifl ength } \triangle 0 \\
\partial 1=20 P^{*} \text { length }-1=2 & \text { if } 10 \text { Cength } 30 \\
1 & \text { if } 30 \text { Cength }\end{array}$ & Checa and Prendergast [16] \\
\hline ECs & Vessel rate of growth & $\begin{array}{cc}-\left(33=5 p^{*} S p 33\right. & \text { if } S G \\
0 & \text { if } S G\end{array}$ & Checa and Prendergast \\
\hline ECs & Vessel direction of growth & $\begin{array}{l}\text { Random direction with } \mathrm{p}^{1 / 4} 0.4 \\
\text { Previous direction with } \quad \mathrm{p}^{1 / 4} 0.2 \\
\text { VEGF direction with } \quad \mathrm{p}^{1 / 4} 0.4\end{array}$ & Checa and Prendergast [16] \\
\hline
\end{tabular}

includes individual cell processes like proliferation, migration, differentiation and apoptosis and simultaneously uses consecutive lattice points to represent capillaries. This model was also applied to a simplified scaffold for bone tissue engineering relating the number of cells initially seeded into the scaffold to the rate of vascularization and the penetration of the vascular network. The effect of the mechanical load magnitude applied to the scaffold on bone formati on and capillary growth was also studied.

The mechanobiological model of Checa and Prendergast was applied to a scaffold of regular geometry [17], i.e. constant pore size and pore distribution. However, depending on the fabrication method, scaffolds do not usually have a controlled regular morphology, e.g. Calcium Phosphate (CaP) based scaffolds, where the macroporosity is achieved mixing the material with foaming agents. In silico studies based on micro Computed Tomography (CT) images of scaffolds have shown that the morphology of constructs has an important influence on the local mechanical stimuli sensed by cells attached to the internal walls of the material [18-22] . According to the pore distribution the microscopic stimuli within the material can be much higher or lower than the macroscopic stimuli applied to the scaffold [22] .

The objective of this study was to investigate angiogenesis and tissue differentiation within the pores of a CaP scaffold with irregular morphology. To achieve this, the method presented in Checa and Prendergast [16] was used to simulate the processes. We hypothesized that the vascular network formed and the distribution of differentiated tissue are critically affected by the morphology of the scaffold.

\section{Materials and methods}

\subsection{Material sample and computed reconstruction}

A cylindrical section of $1 \mathrm{~mm}$ diameter and $0.6 \mathrm{~mm}$ height of a CaP based glass ceramic scaffold with irregular porosity $(21 \%)$ and morphology studied previously [22] was used ( Fig. 1). The detailed methodology of computed reconstruction and meshing was described in Sandino et al. [22] and is summarized in Fig. 2. Briefly, micro CT images of voxel size $7.8 \times 7.8 \times 12.2 \mathrm{~mm}^{3}$ were obtained to discretize the porous space from the solid material phase. A 3D solid reconstruction was performed using Mimics (Materialise, Belgium) and the mesh for the Finite Element (FE) model was made using Patran (MSC Software, CA-USA). The FE mesh of the section used in this study has 174,015 10-node tetrahedral elements in the solid phase and 24,907 in the pore phase.

\subsection{FE model and computation of mechanical stimulus}

The mechanical stimulus regulating cell differentiation was defined as $\mathrm{S} 1 / 4 \mathrm{~g} / \mathrm{a} \mathrm{p} / \mathrm{b}$, where $\mathrm{g}$ was the octahedral shear strain, $\mathrm{n}$ was the fluid velocity (in $\mathrm{mm} / \mathrm{s}$ ), and $a$ and $b$ were empirical parameters equal to 0.0375 and $0.003 \mathrm{~mm} / \mathrm{s}$ respectively [3]. S was computed from the FE model using a poroelastic formulation in Abaqus 6.8.3 (Simulia, RI-USA). Unconfined compressive strain in the scaffold was simulated fixing the nodes at the bottom of the sample and applying displacement to the nodes at the top. Null pressure at the outside of the interconnected pores was imposed allowing fluid flow across the boundary.

\subsection{Angiogenesis and cell differentiation algorithms}

Angiogenesis and cell differentiation were simulated within the interconnected pores of the scaffold. This domain was divided in equidistant points (lattice points) forming an orthogonal grid. Each point represented the volume space that can be occupied by one cell and its extracellular matrix. The distance between each point was $10 \mathrm{~mm}$. Cell activity was mimicked in the lattice points following Bernoulli probability distributions; it means that an event occurred with a probability p or not with a probability $\left(\begin{array}{ll}1 & -p\end{array}\right)[16]$. A schematic flow chart of the methodology is showed

Table 2

Material properties used in the FE models.

\begin{tabular}{|c|c|c|c|c|c|c|}
\hline & Fibrous tissue $^{a}$ & Cartilage $^{\text {a }}$ & Immature bone ${ }^{a}$ & Mature bone ${ }^{a}$ & Granulation tissue $^{a}$ & CaP glass \\
\hline Young's modulus (MPa) & 2 & 10 & 1000 & 6000 & 0.2 & $455^{b}$ \\
\hline Poisson's ratio & 0.167 & 0.167 & 0.3 & 0.3 & 0.167 & $0.33^{b}$ \\
\hline Permeability $\left(\mathrm{m}^{4} / \mathrm{Ns} \times 10^{-14}\right.$ ) & 1 & 0.5 & 10 & 37 & 1 & 1 \\
\hline Bulk modulus grain (MPa) & 2300 & 3700 & 13920 & 13920 & 2300 & 13920 \\
\hline Bulk modulus fluid (MPa) & 2300 & 2300 & 2300 & 2300 & 2300 & 2300 \\
\hline
\end{tabular}

a Lacroix et al. [5].

b Lacroix et al. [19]. 
Table 3

Simulations performed using different compressive strain magnitudes and MSCs initialization modes.

\begin{tabular}{lcc}
\hline Simulation & Total compressive strain (\%) & MSCs initialization \\
\hline 1 & 0.5 & In vitro seeding \\
2 & 0.5 & In vivo \\
3 & 1.0 & In vitro seeding \\
4 & 1.0 & In vivo \\
\hline
\end{tabular}

in Fig. 2 and the parameters and probabilities used in this study are summarized in Table 1 . Since the degradation rate of phosphate glasses is very slow (less than $2 \%$ of weight in 80 days [23] ), scaffold degradation was not simulated and the mesh and the lattice did not change. Each iteration represented one day.

\subsection{Mesenchymal stem cells (MSCs) and endothelial cells (ECs) initialization}

Two different cases of MSCs initialization were simulated. In the first case, called in vitro seeding, an in vitro MSCs seeding previous to the scaffold implantation was assumed, consequently MSCs were attached to the walls of the material at the beginning of the simulation. In the second case, called in vivo colonization, no previous in vitro seeding was performed, then it was assumed that MSCs were initially located at the outside of the interconnected pores.

At the beginning of the simulation, each lattice point at the surface of the scaffold, or at the outside of the interconnected pores according to the case, had a probability for locating a cell. In the first case, this probability was based on the mechano-regulatory stimulus $\mathrm{S}$ corresponding to the lattice point and had a normal distribution with mean 1.5 and variance 0.45 . These mean and variance were fixed so that the mean value of the initial number of MSCs corresponded to an in vitro seeding efficiency of $40 \%$ [24]. In the second case, the probability was 0.3 . Initially, every MSC had a maturation age of 1 and it was increased by 1 each iteration.

For both cases of MSCs initialization, ECs were located at the outside of the interconnected pores; each unoccupied lattice point had a probability of 0.1 of locating a cell.

\subsection{MSCs differentiation}

Every MSC with a maturation age higher than 5 differentiated with a certain probability. If a cell differentiated, the phenotype was assigned according to the mechano-regulatory stimuli $\mathrm{S}$ corresponding to the specific location of the cell. When $\mathrm{S}$ corresponded to osteoblastic differentiation, oxygen supply was taken into account. If there were one or more ECs within the surrounding space corresponding to the oxygen diffusion distance around the MSC, the cell differentiated into an osteoblast. Else, it differentiated into a chondrocyte.

\subsection{Cell apoptosis}

Any osteoblast, chondrocyte or fibroblast located in a lattice point whose mechanical stimulus did not support that cell phenotype led to apoptosis with a probability $\mathrm{p}$. Additionally, if the stimulus $\mathrm{S}$ was higher than 6 , the cell became apoptotic.

\subsection{Cell proliferation}

Every cell proliferated with a probability p. If a cell proliferated, mitosis occurred and the cell was replaced by two daughter cells. The new cells occupied two points selected at random within the original location and its empty neighbouring locations.

\subsection{Cell migration}

Every MSC and fibroblast migrated; it means that they moved to any empty point beside them. The new position was selected at random. If there were no unoccupied neighbouring locations then migration stopped. Since osteoblasts and chondrocytes are less motile, migration was not simulated.

\subsection{Angiogenesis}

Blood vessels were represented as a continuum sequence of attice points occupied by ECs. For the angiogenesis process, the direction and the rate of growth of each vessel, and its probability of branching were defined. The direction of growth was assigned according to three cases. In the first case the direction was assigned randomly, in the second case the direction was the same than the previous growth direction and in the last case the vessel grew towards the region with the highest concentration of chondrocytes under a mechanical stimulus corresponding to osteoblasts (representing the VEGF influence). The rate of growth was defined as a function of the mechanical stimulus at the front of the vessel. The probability of branching was assigned according to the length of the vessel. Each vessel grew according to its defined variables. Functions and probabilities are shown in Table 1.

\subsection{Material properties}

After each iteration, the element material properties (Young's modulus, Poisson's ratio and permeability) were computed as an average of the properties for the tissue phenotypes predicted in the lattice points within the element in the last 10 iterations [4]. Material properties are shown in Table 2.

\subsection{Simulations}

In order to study the effects of the total strain magnitude and the MSCs seeding mode on the tissue formation process, four cases were simulated, varying the compressive strain $(0.5 \%$ and $1 \%)$ or varying the initial cell seeding ( in vitro and in vivo), see Table 3 .

\section{Results}

The growth of vessels and cell differentiation over time in a cross section through the center of the scaffold for the simulation $1(0.5 \%$ of total compression and in vitro MSCs seeding) is shown in Fig. 3. Most of the capillaries started growing from the periphery of the sample at iteration 1 and were blocked by the walls of the scaffold, with only a few vessels branching. Therefore, pores located at the surface of the sample were well vascularized but pores at the center were not. MSCs proliferated and started filling the scaffold during the first iterations. A rapid differentiation of cells into osteoblasts and chondrocytes was predicted between iterations 10 and 20 when the first stem cells achieved the maturation age. At iteration 20 the pores were filled with MSCs, osteoblasts and chondrocytes. At iteration 30 most of the stem cells had differentiated and after 100 iterations the dynamics of proliferation, differentiation, and apoptosis was stable.

The evolution of the effective Young's modulus during the simulation and the mechano-regulatory stimulus $\mathrm{S}$ (as opposed to tissue type in Fig. 3 ) influencing each lattice point at the first and last iterations are shown in Fig. 4. At iteration 1 it was not possible
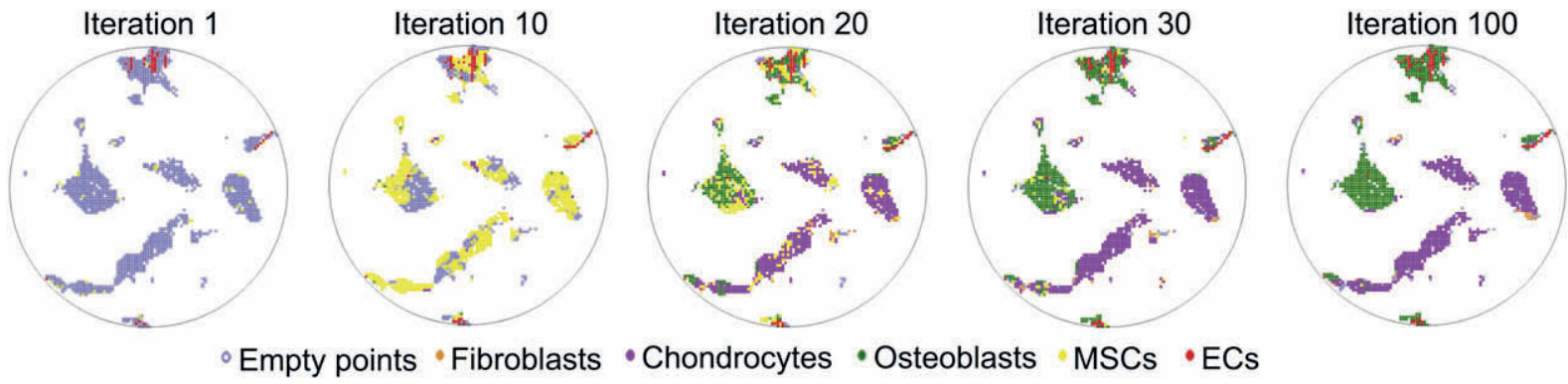

Fig. 3. Evolution of cell distribution over time in a cross section at the center of the sample in simulation $1(0.5 \%$ of total strain and

in vitro MSCs seeding). Vessels grew mainly in the pores at the periphery of the sample. At the end of the simulation osteoblasts were predicted in the vascularized pores and chondrocytes in the non-vascularized ones. 


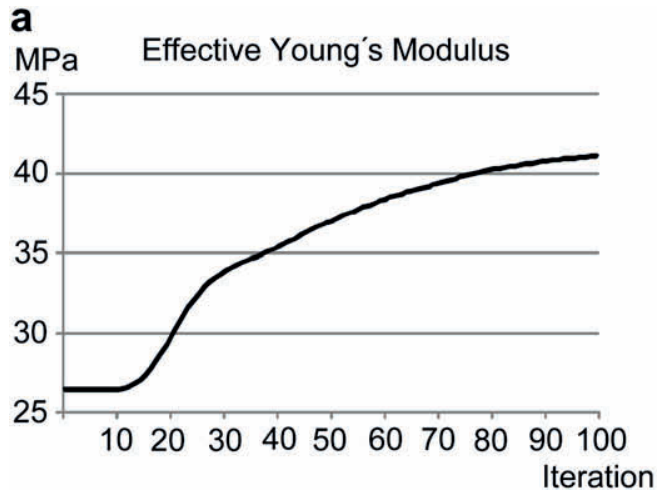

b

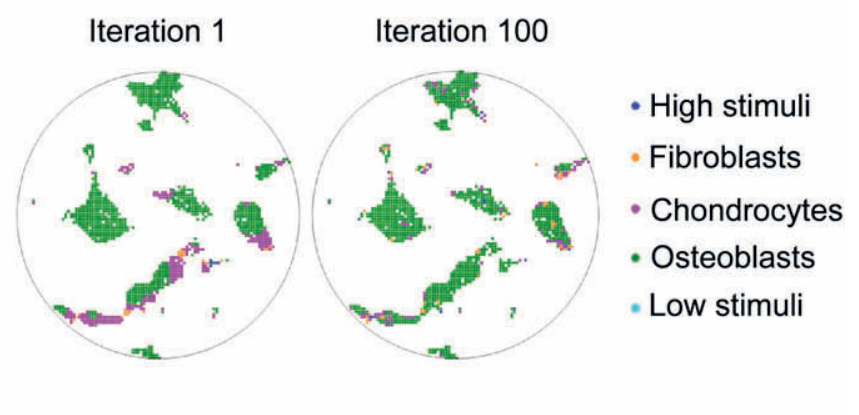

Fig. 4. (a) Effective Young's modulus (Reaction force/Area \$Strain) of the sample ove center of the sample at iterations 1 and 100 in simulation 1 (0.5\% of total strain and modulus increased and the mechano-reculatory stimulus distribution became uniform.

ime. (b) Mechano-regulatory stimuli $\mathrm{S}$ affecting cell differentiation in a cross section at the in vitro MSCs seeding). When differentiated cells started filling the pores, the effective Young's

to find a direct relation between pore size and stimuli, since simultaneous stimuli corresponding to bone, cartilage and fibrous tissue were calculated in most of the pores. Then cells started differentiating, therefore the stiffness of the sample started increasing. Throughout the simulation the effective Young's modulus increased from 26.44 MPa to 41.14 MPa and most of the mechanical stimuli predicted were those of bone formation $(70 \%$ of pore volume). Although most of stimuli corresponded to bone formation, different types of tissue were predicted within the pores of the sample ( Fig. 5). $70 \%$ of pore volume corresponded to bone stimuli but, because of the lack of oxygen, only $40 \%$ of the cells in this pore volume differentiated into osteoblasts. At the end of the simulation $40 \%$ of the pore volume was filled with osteoblasts, $40 \%$ with chondrocytes and 3\% with fibroblasts. Osteoblasts were predicted in the pores located at the periphery of the sample (well vascularized regions), whereas chondrocytes were predicted in the internal pores (poor vascularized ones) and fibroblasts were predicted in the narrow regions where there was a high fluid flow.

The vascular network formation was not very sensitive to the MSCs seeding mode. Similar distributions of vessels were predicted whether an in vitro seeding or an in vivo colonization was simulated. The MSCs seeding mode did not have either an evident effect on the distribution of tissue at the end of the simulation. Nevertheless, when previous in vitro seeding was simulated, the cells migrated and proliferated faster than when in vivo colonization was simulated.

For the magnitudes of mechanical strain studied $(0.5 \%$ and $1 \%$ of total deformation), there was not a noticeable effect on angiogenesis. Similar vessel networks were observed in both cases of strain. Nevertheless different types of tissues were observed within the pores of the sample according to the mechanical strain applied (Fig. 6). When $0.5 \%$ of deformation was simulated osteoblasts were predicted in well vascularized regions and chondrocytes in poor vascularized ones. When the total strain was increased to $1 \%$ of deformation, chondrocytes were predicted in both, internal and external pores. In the case of high stain (1\% of deformation) $11 \%$ of the pore volume was filled with osteoblasts, $59 \%$ with chondrocytes, and $8 \%$ with fibroblasts. In this case the Effective Young's modulus of the sample increased only to $28.19 \mathrm{MPa}$, instead of $41.14 \mathrm{MPa}$ (when $0.5 \%$ of deformation was imposed).

\section{Discussion}

In this work we set out to investigate vascularization and tissue formation inside a CaP scaffold of $21 \%$ of nterconnected porosity with irregular morphology. It was predicted that scaffold walls blocked blood vessel growth and consequently, most of the vessels did not reach the center of the sample and a vascular network was mainly formed in the external pores. Therefore, when a compressive strain of $0.5 \%$ was applied, $70 \%$ of the pore volume was under a mechanical stimulus favorable for bone formation; however, because of the lack of oxygen in some regions, MSCs differentiated into chondrocytes instead of osteoblasts and only $40 \%$ of bone was formed. In the scaffold with regular morphology studied by Checa and Prendergast [17] full vascularization was achieved and consequently cell differentiation depended mainly on the mechanical stimuli. This difference suggests that in terms of the architecture of the samples, it is necessary to ensure a structure that not only has high porosity and interconnectivity, but also that the space a

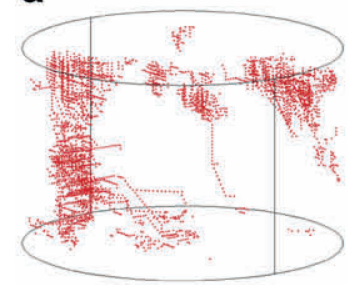

b

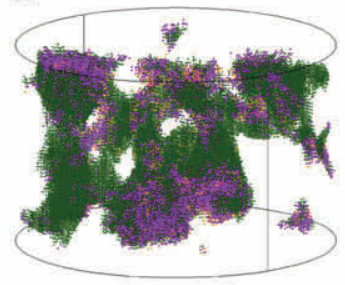

- Empty points - Fibroblasts - Chondrocytes - Osteoblasts MSCs ECs

C

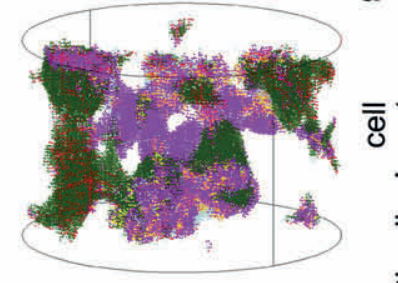

d

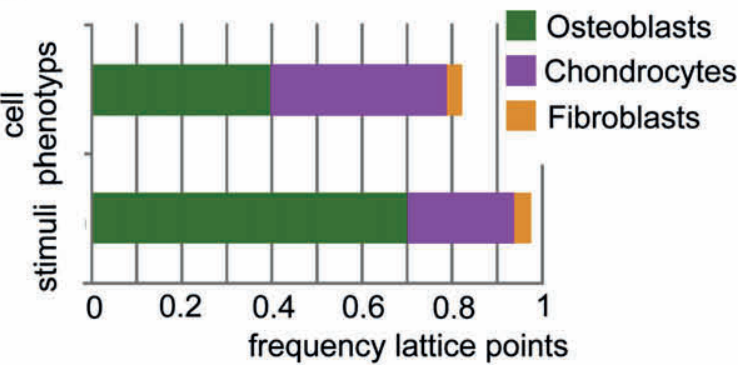

Fig. 5. Contribution of angiogenesis and mechano-regulatory stimuli to cell differentiation in simulation $1(0.5 \%$ of total strain and

in vitro MSCs seeding) after 100 iterations. (a) Vascular network. (b) Stimuli distribution. (c) Cell distribution. (d) Frequency of osteoblasts, chondrocytes and fibroblasts predicted compared with the frequency of mechanoregulatory stimuli affecting cell differentiation at the end of the simulation. $70 \%$ of the pore volume was influenced by bone stimuli but, because of the lack of oxygen, only $40 \%$ of the pore volume was filled with osteoblasts. 

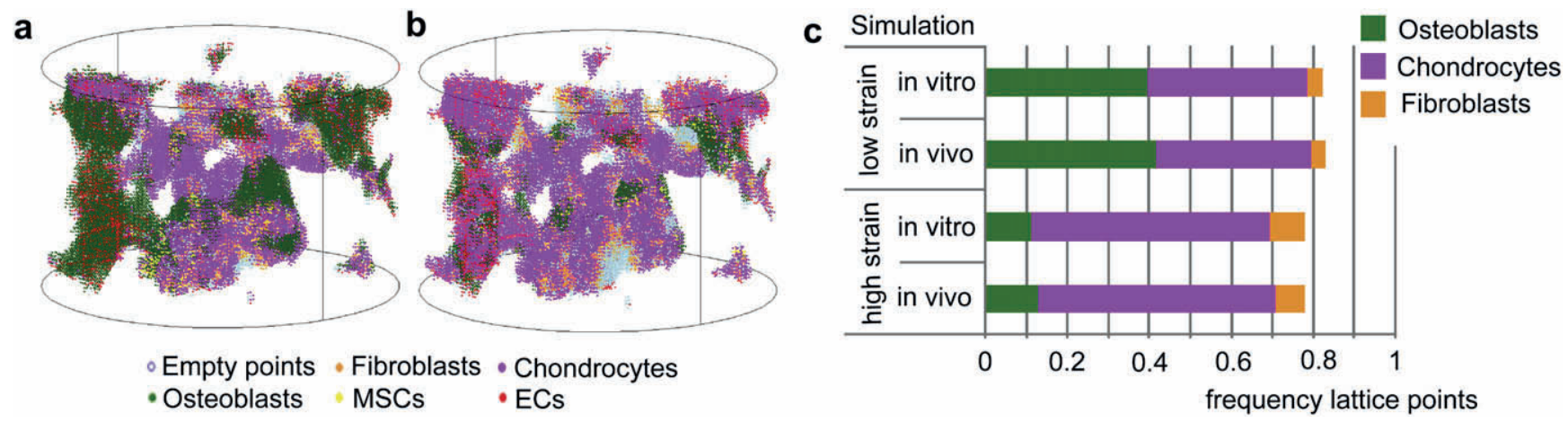

Fig. 6. Effect of the compressive strain and the MSC initialization mode on the distribution of differentiated cells after 100 iterations. (a) Cell distribution when $0.5 \%$ of deformation was applied (simulation 1). (b) Cell distribution when 1\% of deformation is applied (simulation 3). (c) Frequency of osteoblasts, chondrocytes and fibroblasts predicted at the end of the simulations. Similar cell distributions were predicted at the end of the simulation when the MSC initialization mode was changed. The frequency of osteoblasts and chondrocytes predicted at the end of the simulation decreased and increased respectively when the compressive strain was increased.

between pores allows vessels to reach the center of the scaffolds and form a vascular network.

The magnitude of the mechanical strain applied to the sample had an important effect on tissue differentiation. When the compressive strain was increased from $0.5 \%$ to $1 \%$, osteogenesis decreased from $40 \%$ to $15 \%$. This result showed that the sensitivity of cell differentiation to small changes in the magnitude of the strain should be considered in in vivo experiments where bone chambers are used to apply controlled mechanical stimuli. Additionally, when the material is implanted directly into bone, the performance of the scaffold can change depending on the implantation site because of the variation of the loads and therefore the mechanical stimuli along the bone.

MSC seeding conditions did not affect the final pattern of differentiated tissue. Similar results were obtained when in vivo or in vitro MSC seeding were simulated. The effect of ncreasing the number of cells seeded in the scaffold was not studied. Checa and Prendergast [17] predicted that when the number of seeded cells was increased, the space for angiogenesis was decreased and a lower number of vessels was predicted. In this study, most of the vessels were formed at iteration one, then they were blocked and the rest of the space was filled with cells proliferating. When in vivo seeding was simulated, similar results than Checa and Prendergast [17] were obtained; differentiation occurred first in the external pores and then in the internal ones, the tissue formation was slower than in the case of in vitro seeding.

Reconstruction of the scaffold was obtained from micro CT images of $7.8 \times 7.8 \times 12.2 \mathrm{~mm}^{3}$, therefore pores smaller than this size were not detected by the scanner. Additionally, just one cylindrical section of $1 \mathrm{~mm}$ diameter and $0.6 \mathrm{~mm}$ height of one scaffold was analyzed. Nevertheless, taking into account the computational cost to simulate a complete scaffold, this section was considered sufficient to study the effect of architecture in angiogenesis and tissue differentiation.

The algorithm of mechanoregulation has been validated for bone healing [25]. A study of the parameters used in the algorithm of angiogenesis was done and its limitations were discussed in detail by Checa and Prendergast [16]. Here, the base line model parameters were used. Some parameters that are regulated by growth factors were simulated as random, for instance the differentiation rate, considered constant. The fact that cells do not migrate to regions where there is no oxygen was not considered by the algorithm, so pores in the center of the sample, where angiogenesis did not occur, could be actually empty instead of being occupied by chondrocytes. The results of this study were consistent with the in vivo observations of Del Valle et al. [26] for the macroporous CaP scaffolds and Jones et al. [27] for the hydroxyapatite scaffolds. Del Valle et al. [26] found that blood vessels were present near the surface of the cement and that bone growth occurred mainly in the peripheral pores. They affirm that the lack of bone tissue penetration in the central part of the implant was due to the limited interconnectivity between adjacent pores. Jones et al. [27] showed that approximately $40 \%$ of pores occupied by bone tissue were near the surface and this fraction dropped within $1 \mathrm{~mm}$ of the periphery where most pores were free of bone ingrowth. The use of sample specific models to compare in vivo and in silico results can be useful to improve the development of this kind of models. Bone ingrowth into biomaterials in in vitro or in vivo experiments can be assessed using micro CT images [28,29] .

The mechanoregulation model of Prendergast et al. [3] predicts fibrous tissue formation when the mechano-regulatory stimuli is higher than 3. However, cells can undergo apoptosis under high levels of strain [30]. In order to take this effect into account, a maximum limit was included in the model, if the mechanical stimuli were higher than 6 no tissue was differentiated. In the studied scaffold, the percentage of pore volume affected by stimuli out of the physiological range, being resorption or apoptosis, increased from $17 \%$ to $22 \%$ when the mechanical strain was increased from $0.5 \%$ to $1 \%$ of compression. This increment was low (only 5\%), because the magnitude of the mechano-regulatory stimuli $S$ in most of regions where apoptosis occurs was much higher than the maximum limit applied. Consequently, the obtained results were not sensitive to the assumed value of 6 for the limit between fibrous tissue formation and apoptosis.

At the beginning of the simulation, there was not a clear correlation between stimuli and pore size or pore location, different stimuli were observed within every pore. It was studied previously [22] that the octahedral strain depends on the thickness of the scaffold walls and the fluid velocity on the pore interconnectivity making the distribution of the mechano-regulatory stimuli not evident. When tissue growth was simulated, the scaffold stiffness increased and consequently stress and strain were better distributed across the material. At the end of the simulation the stimuli were mostly in favor of bone formation. Because the mechanical stimuli changed over time due to the formation of new tissue, investigations on the effect of mechanical loading on the distribution of mechanical stimuli within an empty scaffold help to determine the behavior of the scaffold, but they are not enough to predict the success of the material once tissue starts to differentiate. Consequently, a dynamical analysis is necessary to understand the contribution of the geometry and to predict the mechanoregulation within scaffolds for bone tissue engineering. 


\section{Conclusions}

This study provided information on the dynamics of mechanical stimuli, angiogenesis and tissue differentiation within the interconnected pores of a CaP scaffold with irregular morphology caused by mechanical compressive strain. Different types of tissues were predicted into the scaffold depending not only in the mechanical stimuli distribution but also in the blood vessel network formation. Osteogenesis was not predicted in most of the pores at the center of the scaffold. This information contributed to the understanding of the effect of angiogenesis and mechanical stimuli on tissue differentiation within the CaP scaffolds. Such kind of studies combined with in vivo and in vitro experiments are essential to the improvement of scaffold development.

\section{Acknowledgments}

The authors acknowledge funding from the Government of Catalonia, the European Commission through the SmartCaP project (NMP3-CT2005-013912) and Science Foundation Ireland. Hanifeh Khayyeri is acknowledged for her help in the discussion of the results.

\section{Appendix}

Figure with essential color discrimination. Most of the figures in this article have parts that are difficult to interpret in black and white. The full colour images can be found in the on-line version, at doi:10.1016/j.biomaterials.2009.11.063

\section{References}

[1] Carter D, Blenman P, Beaupre ' G. Correlations between mechanical stress history and tissue differentiation in initial fracture healing. J Orthop Res 1988;6(5):736-48.

[2] Claes L, Heigele C. Magnitudes of ocal stress and strain along bony surfaces predicts the course and type off racture healing. J Biomech 1999;32(3): 255-66.

[3] Prendergast PJ, Huiskes R, Søballe K. Biophysical stimuli on cells during tissue differentiation at implant interfaces. J Biomech 1997;30(6):539-48.

[4] Lacroix D, Prendergast PJ, Li G, Marsh D. Biomechanical model to simulate tissue differentiation and bone regeneration: application to fracture healing. Med Biol Eng Comput 2002;40(1):14-21.

[5] Lacroix D, Prendergast PJ. A mechano-regulatory model for tissue differentiation during fracture healing: analysis of gap size and loading. J Biomech 2002;35(9):1163-71.

[6] Isaksson H, Wilson W, Donkelaar CCv, Huiskes R, Ito K. Comparison of biophysical stimuli for mechano-regulation of tissue differentiation during fracture healing. J Biomech 2006;39(8):1507-16.

[7] Hayward LNM, Morgan EF. Assessment of a mechano-regulation theory of skeletal tissue differentiation in an in vivo model of mechanically induced cartilage formation. Biomech Model Mechanobiol 2009;. doi:10.1007/s10237009-0148-3.

[8] Liu X, Niebur GL. Bony ingrowth into a porous coated implant predicted by a mechano-regulatory tissue differentiation algorithm. Biomech Model Mechanobiol 2007;7(4):335-44

[9] Geris L, Andreykiv A, Oosterwyck HV, Sloten JV, Keulen Fv, Duyck J, et al. Numerical simulation of tissue differentiation around loaded titanium implants in a bone chamber. J Biomech 2004;37(5):763-9.
[10] Geris L, Vandamme K, Naert I, Sloten JV, Duyck J, Oosterwyck HV. Application of mechanoregulatory models to simulate peri-implant tissue formation in an in vivo bone chamber. J Biomech 2008;41(1):145-54.

[11] Kelly DJ, Prendergast PJ. Prediction of the optimal mechanical properties for a scaffold used in osteochondral defect repair. Tissue Eng 2006;12(9):2509-19.

[12] Pe'rez MA, Prendergast PJ. Random-walk models of cell dispersal included in mechanobiological simulations of tissue differentiation. J Biomech 2007; 40(10):2244-53.

[13] Byrne DP, Lacroix D, Planell JA, Kelly DJ, Prendergast PJ. Simulation of tissue differentiation in a scaffold as a function of porosity, Young's modulus and dissolution rate: application of mechanobiological models in tissue engineering. Biomaterials 2007;28(36):5544-54.

[14] Khayyeri H, Checa S, Ta "gil M, Prendergast PJ. Corroboration of mechanobiological simulations of tissue differentiation in an in vivo bone chamber using a lattice-modeling approach. J Orthopaed Res 2009;27(12):1659-66.

[15] Geris L, Gerisch A, Sloten JV, Weiner R, Oosterwyck HV. Angiogenesis in bone fracture healing: a bioregulatory model. J Theor Biol 2008;251(1):137-58.

[16] Checa S, Prendergast PJ. A mechanobiological model for tissue differentiation that includes angiogenesis: a lattice-based modeling approach. Ann Biomed Eng 2009;37(1):129-45.

[17] Checa S, Prendergast PJ. Effect of cell seeding and mechanical loading on vascularization and tissue formation inside a scaffold: a mechano-biological model using a lattice approach to simulate cell activity. J Biomec, in press. doi:10.1016/j.jbiomech.2009.10.044.

[18] Cioffi M, Boschetti F, Raimondi MT, Dubini G. Modeling evaluation of the fluiddynamic microenvironment in tissue-engineered constructs: a micro-CT based model. Biotechnol Bioeng 2006:93(3):500-10.

[19] Lacroix D, Cha ^teau A, Ginebra MP, Planell JA. Micro-finite element models of bone tissue-engineering scaffolds. Biomaterials 2006;27(30):5326-34.

20] Milan JL, Planell JA, Lacroix D. Computational modelling of the mechanical environment of osteogenesis within a polylactic acid-calcium phosphate glass scaffold. Biomaterials 2009;30(25):4219-26.

[21] Porter B, Zauel R, Stockman H, Guldberg R, Fyhrie D. 3-D computational modeling of media flow through scaffolds in a perfusion bioreactor. J Biomech 2005;38(3):543-9.

[22] Sandino C, Planell JA, Lacroix D. A finite element study of mechanical stimuli in scaffolds for bone tissue engineering. J Biomech 2008;41(5):1005-14.

[23] Navarro M, Ginebra MP, Cle 'ment J, Martı 'nez S, Avila G, Planell JA. Physicochemical degradation of titania-stabilized soluble phosphate glasses for medical applications. J Am Ceram Soc 2003;86(8):1345-52.

[24] Lopez-Heredia MA, Sohier J, Gaillard C, Quillard S, Dorget M, Layrolle P. Rapid prototyped porous titanium coated with calcium phosphate as a scaffold for bone tissue engineering. Biomaterials 2008;29(17):2608-15.

[25] Isaksson $\mathrm{H}$, Donkelaar CCV, Huiskes $\mathrm{R}$, Ito K. Corroboration of mechanoregulatory algorithms for tissue differentiation during fracture healing: comparison with in vivo results. J Orthop Res 2006;24(5):898-907.

[26] Del Valle S, Min o N, Muñ os F, Gonza'lez A, Planell JA. In vivo evaluation of an injectable macroporous calcium phosphate cement. J Mater Sci Mater Med 2007;18(2):353-61.

[27] Jones CA, Arns CH, Sheppard AP, Hutmacher DW, Milthorpe BK, Knackstedt MA. Assessment of bone ingrowth into porous biomaterials using micro-CT. Biomaterials 2007;28(15):2491-504.

[28] Porter BD, Lin ASP, Peister A, Hutmacher D, Guldberg RE. Noninvasive image analysis of $3 \mathrm{D}$ construct mineralization in a perfusion bioreactor. Biomaterials 2007:28(15):2525-33.

[29] Lenthe GHv, Hagenmu "ller H, Bohner M, Hollister SJ, Meinel L, Mu "Iler R. Nondestructive micro-computed tomography for biological imaging and quantification of scaffold-bone integration in vivo. Biomaterials 2007;28(15): 2479-90.

[30] Kearney EM, Prendergast PJ, Campbell VA. Mechanisms of strain mediated mesenchymal stem cell apoptosis. J Biomech Eng 2008;130(6):061004.

[31] Isaksson H, Donkelaar CCv, Huiskes R, Ito K. A mechano-regulatory bonehealing model incorporating cell-phenotype specific activity. J Theor Biol 2008;252(2):230-46.

[32] Carmeliet $P$, Jain RK. Angiogenesis in cancer and other diseases. Nature 2000;407(6801):249-57.

[33] Appedu PA, Shur BD. Molecular analysis of cell surface b-1,4-galactosyltransferase function during cell migration. Proc Natl Acad Sci U S A 1994;91:2095-9. 